\title{
Educación del Trabajo Social en Chile: 90 años de Historia*
}

\author{
Educação do Trabalho Social no Chile: 90 anos de história \\ Social work education in Chile: 90 years of history \\ Paula Vidal Molina**
}

\begin{abstract}
Resumen - El artículo aborda, desde una mirada panorámica, la historia de modificaciones sufridas en la formación del Trabajo Social en Chile. Señala las orientaciones de las escuelas entre los ciclos 1925-1960, 19601973 y 1973-2012, a través de un estudio de caso: la Escuela de Servicio Social Dr. Lucio Córdova de la Universidad de Chile. La Universidad pública más importante de Chile y fuertemente golpeada por las reformas estructurales realizadas por la Dictadura Militar. Realizada la revisión de las orientaciones de las escuelas, se observa la estrecha relación existente entre procesos históricos y la formación de 90 años del Trabajo Social chileno.

Palabras clave: Trabajo Social; historia de Chile, formación académica, Universidad de Chile.
\end{abstract}

Resumo - O artigo aborda, a partir de uma perspectiva panorâmica, a história das modificações sofridas na formação do Trabalho Social no Chile. Assinala as orientações das escolas entre os ciclos 1925-1960, 1960-1973 e 1973-2012, por meio de um estudo de caso: a Escola de Serviço Social Dr. Lucio Córdova, da Universidade do Chile, universidade pública mais importante do Chile e fortemente atingida pelas reformas estruturais realizadas pela Ditadura Militar. Realizada a revisão das orientações das escolas, se observa a estreita relação existente entre processos históricos e a formação de 90 anos do Trabalho Social chileno.

Palavras-chave: Trabalho Social; história do Chile; formação acadêmica; Universidade do Chile.

\begin{abstract}
The article addresses, from a panoramic view, the history of modifications suffered in the formation of social work in Chile. It points out the orientations of the schools during the cycles of 1925-1960, 19601973, and 1973-2012 through a case study: the Dr. Lucio Córdova School of Social Service of the University of Chile, the most important public
\end{abstract}

\footnotetext{
* Publicação original na revista italiana Quaderni del Csal, Anno VII, Numero 13, Luglio 2015, Numero speciale: Social work around the world. Colors and shapes in a complex mosaic.

** Doctora en Servicio Social por la Universidad Federal de Rio de Janeiro. Brasil. Actualmente se desempeña como Académica en la Universidad de Chile. E-mail: pvidal@u.uchile.com. ORCID: https://orcid.org/0000-00029036-3766.
} 


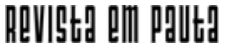

\} EDUCACIÓN DEL TRABAJO SOCIAL EN CHILE - MOLINA, P. V. \}

DOI: $10.12957 /$ REP.2019.45217

University in Chile and strongly hit by the structural reforms carried out by the military dictatorship. After the revision of the orientations of the schools, there can be seen the close relationship between historical processes and the formation of 90 years of Chilean social work.

Keywords: social work; Chilean history; academic training; University of Chile.

\section{Orígenes del Trabajo Social chileno: 1925}

Para comprender los cambios ocurridos en 90 años de vida del Trabajo Social chileno, debemos entender la historia social del país. En ese sentido, la profesión nació ligada a los desafíos sociales, culturales y políticos de Chile de mediados de la década de 1920.

Los estudios historiográficos más recientes sobre el Trabajo Social Chileno ${ }^{1}$ concuerdan con el origen secular de la profesión en Chile, pues la primera escuela de Servicio Social, Dr. Alejandro del Río (pionera en Chile y América Latina) abrió sus puertas en 1925. Se vinculó inmediatamente a la atención sanitaria, a través de la Junta Nacional de Beneficencia (correspondiente al actual Ministerio de Salud), con un carácter científico, propio de la época. En ese sentido, la influencia moderna se expresaba en la orientación de la malla curricular para formar profesionales con una orientación distinta a la caritativa y moral, bien se expresa en la revista de Servicio Social del año 1928:

el concepto de caridad, que tanto daño y atraso social ha llevado a la sociedad chilena, el que ha permitido que las mayores estigmatizaciones de su juventud, de sus mujeres, de sus niños, de sus trabajadores, de las familias de escasos recursos económicos, de sus obreros, se hayan expandido y muchas veces ramificado; no entrará jamás en el vocabulario del Servicio Social, ya que éste lucha por su contrario, por una asistencia organizada y justa que le devuelva a cada ser humano su propio valor. (CARDENAS apud MATUS et al., 2004, p. 39).

Además, según Sand:

Es dentro de este vasto dominio donde se coloca el Servicio Social, quien se contrapone a la caridad y la filantropía y se distingue en la asistencia por su carácter científico y sistemático, por su cuidado en la investigación de las causas, por la extensión de su campo de estudio y de acción. (SAND apud MATUS et al., 2004, p. 46).

\footnotetext{
"La Reinvención de la Memoria" (MATUS et al., 2004) y "El cuerpo y la sangre de la política. La construcción histórica de las visitadoras sociales. Chile. 1887-1940" (ILLANES; MARÍA, 2007).
} 


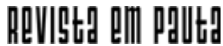

\} EDUCACIÓN DEL TRABAJO SOCIAL EN CHILE - MOLINA, P. V. \}

DOI: $10.12957 /$ REP.2019.45217

Prontamente, en 1929, se abrió la segunda escuela - Elvira Matte -, de carácter religioso y ligada a la Pontificia Universidad Católica (PUC) de Chile. Las primeras profesionales egresadas de ambas escuelas debieron enfrentar los efectos desastrosos en los sectores sociales más pobres y desprotegidos del país, como secuela del derrumbe de la economía chilena debido a la crisis mundial de 1930. Durante esta década, el Partido Radical (ubicado en el centro político), el Partido Socialista y el Partido Comunista acercaron posiciones logrando conformar el Frente Popular, alianza política que ganó la presidencia de Chile en 1938 con Pedro Aguirre Cerda a la cabeza y un programa de gobierno democrático y popular (MILOS, 2008).

Este presidente dictó, en 1940, un Decreto Supremo, que permitió crear las Escuelas de Servicio Social de Santiago, Concepción y Temuco. Estas dependieron del Ministerio de Educación Pública, pero en 1948 fueron incorporadas a la Universidad de Chile. Su fundador y primer Director General fue Lucio Córdova (ALVARIÑO, 1965, p. 11), nombre con el cual será bautizada, en la década siguiente, la Escuela de Servicio Social de la Universidad de Chile, localizada en Santiago.

En 1945, la Universidad de Chile fundó también la Escuela de Servicio Social en la ciudad de Valparaíso y, posteriormente, en las ciudades de Antofagasta, Osorno y Arica. Para el año 1971, de las 11 escuelas de Servicio Social que existían en el país, 7 dependían de la Universidad de Chile $^{2}$. Por lo tanto, desde su origen el Trabajo Social chileno fue secular, debido a la influencia y magnitud de escuelas ligadas a la laica y pública Universidad de Chile creada en 1842.

Desde su origen y hasta comienzos de la década de 1960, la formación profesional, especialmente dirigida a mujeres, expresaba la influencia de la sociología positiva y la medicina práctica. Entre los cursos impartidos, estaban: educación cívica, psicología y economía social, higiene y puericultura, atención de enfermos, alimentación y dietética generales, técnica de oficina y estadística, moral, legislación de higiene y beneficencia, legislación del trabajo y de protección a la infancia, puericultura, atención de heridos, alimentación y dietética especial, contabilidad, organización de la beneficencia pública y "el servicio social en sus diversas especializaciones" (ILLANES, 2007, p. 278).

Entre 1925 y 1950, los ámbitos de intervención en los que se desarrolló el Servicio Social chileno fueron de: Salud, Infancia, Educación, Campo (ruralidad), Ciudad, Catástrofes, Laboral y Seguridad Social, lo que se observa en el Gráfico 1, al revisar los contenidos de las 1140 tesis elaboradas por los/las estudiantes de Servicio Social.

\footnotetext{
2 A saber: Escuelas de Servicio Social de la Universidad de Chile, con sedes en las ciudades Arica, Antofagasta, Valparaíso, La Serena, Santiago, Talca, Temuco. Otras escuelas en el país fueron: la Escuela Elvira Matte (dependiente de la PUC de Chile), la escuela Dr. Alejandro del Río, la escuela de la Universidad Católica de Valparaíso y la escuela de la Universidad de Concepción. (CERCEDA et al., 1968, p. 14).
} 


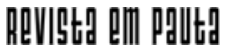

\} EDUCACIÓN DEL TRABAJO SOCIAL EN CHILE - MOLINA, P. V. \}

DOI: $10.12957 /$ REP.2019.45217

Contenidos de las tesis sobreTrabajo Social en Chile hasta 1950

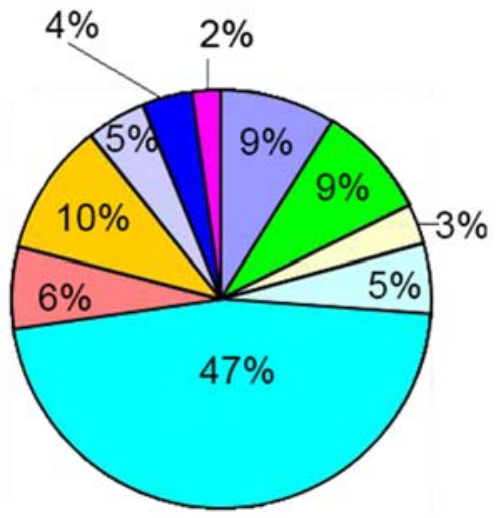

Legislación social y asistencia judicial

Area de la salud y asistencia social

Estudios de la comunidad

Estudios en relación con la familia

Sobre la enseñanza y el desarrollo del Servicio Social

Estudios sobre educación social

Pobreza y problemas socioeconómicos

Estudios sobre asistencia infantil

Estudios sobre cooperativas y bienestar social

Gráfico 1. Fuente: MATUS et al., 2004.

\section{Procesos de cambios del Trabajo Social en Chile: 1960-1973}

Entre 1950 y 1964, las escuelas de Servicio Social de la Universidad de Chile se organizaron y coordinaron a través de la Dirección General de Escuelas de Servicio Social dependiente de la Escuela Dr. Lucio Córdova en Santiago. Ello marcó la influencia de esta escuela no solo en las otras que dependían de la Universidad de Chile, sino también en la más antigua del país, la escuela Dr. Alejandro del Río. Como es sabido, las memorias y tesis de sus estudiantes debían ser aceptadas y reconocidas por la Dirección de la Escuela Dr. Lucio Córdova 3 .

Desde los años 1950 se realizaron algunos cambios en las escuelas de Servicio Social de la Universidad de Chile. En un comienzo los estudios eran de 3 años. A partir de 1964, los estudios académicos pasaron a llevar 4 años, con dedicación al cumplimiento de planes y programas de estudios teóricos y prácticos. Se sumaba un quinto año destinado a desarrollar una investigación para una memoria, conducente al examen de grado y a la obtención del título de Asistente Social. (EDITORIAL, 1966, p. 8).

Hasta el año 1962, las escuelas de la Universidad de Chile impartieron cursos en las áreas de Salud, Salud Mental, Derecho y Legislación, Investigación "práctica" y Métodos de Intervención en Caso, Grupo y

\footnotetext{
${ }_{3}^{3}$ Para fines de 1960, la escuela Dr. Alejandro del Río fue traspasada a la Universidad de Chile, fusionándose con la escuela Dr. Lucio Córdova.
} 


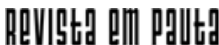

\} EDUCACIÓN DEL TRABAJO SOCIAL EN CHILE - MOLINA, P. V. \}

DOI: $10.12957 /$ REP.2019.45217

Comunidad. Ejemplo de ello fueron los cursos de Higiene, Psicología General, Higiene Mental, Nociones generales de Patología, Enfermería Primeros Auxilios, Alimentación (teórica), Alimentación (práctica), Puericultura (teórica), Puericultura (práctica), Psicología del Niño y Adolescente, Educación Sanitaria, Psicología de la Personalidad, Servicio Social de Colaboración Médica, Derecho: Nociones Generales, Derecho Social, Derecho Procesal, Práctica Legal, Sociología, Conocimientos del Medio Social, Doctrinas Sociales Contemporáneas, Estadística, Investigación Social (teoría), Investigaciones Sociales Prácticas, Nociones de Servicio Social, Método de Servicio Social de Casos, Método de Servicio Social de Grupo, Método de Servicio Social de Organización de Comunidades, Visitas a Instituciones, Normas de Trabajo Práctico, Servicio Social en campos de Aplicación, Organización y Administración de Servicios, Ética Profesional. (Anales de La Facultad de Ciencias Jurídicas y Sociales, 1962). Especialmente los Métodos de Caso, Grupo y Comunidad se mantuvieron durante toda la década de 1960.

Recordemos que el término "Desarrollo de la Comunidad" fue incorporado y posteriormente impulsado desde comienzos de los años 50 por las Naciones Unidas y sus organismos especiales como la Cepal, pero va a ser en 1956 que se acuerda una definición al respecto en estas instancias. Para 1961, en el Servicio Social ya era incorporado y definido en la "Décima Conferencia Internacional de Servicio Social" como

Un proceso consciente, en que las comunidades pequeñas son asistidas por las comunidades más grandes y más avanzadas para lograr mejores niveles de vida social y económica; Ilevando a cabo esto a través de sus esfuerzos locales y por medio de la participación de la comunidad en todas las etapas de selección de metas, movilización de recursos y desempleo de los proyectos hasta que las comunidades logren autonomía. (GARIB, 1969, p. 26).

Se aceptaba que la relación entre los gobiernos, las políticas sociales, la planificación y las comunidades fueran aceleradores del avance de los países subdesarrollados.

Las instituciones con las cuales la escuela Dr. Lucio Córdova contó para que los estudiantes realizaran sus prácticas profesionales durante la década de 1960 fueron organismos del Estado y la empresa privada, a saber: el Servicio Nacional de Salud (en sus consultorios, hospitales, servicios especializados), la Corporación de la Reforma Agraria, el Servicio de Seguro Social, la Dirección General de Asistencia Social, Municipalidades, Industrias como Yarur, Sumar, Bata, Phillips chilena, la Dirección general de Carabineros (Hogares de menores, Juzgados de menores), Corhabit, Escuela especial de lisiados, Promoción Popular, Ejército de Salvación, Escuela especial de ciegos, Politécnico de menores, Ciudades del Niño, Dirección de Pavimentación, Casa Correccional de Mujeres, entre otros (MENDEZ, 1970). 


\section{ReVIStG PMI PDUtD}

\} EDUCACIÓN DEL TRABAJO SOCIAL EN CHILE - MOLINA, P. V. \}

DOI: $10.12957 /$ REP.2019.45217

Asimismo, la necesidad de investigar acerca de la realidad social en la que intervenían los asistentes sociales fue también parte central de la formación universitaria, como el intercambio a nivel internacional. Las visitas, capacitaciones y pasantías en el extranjero fueron realizadas por algunos académicos, con el fin de conocer experiencias y apoyar la formación del cuerpo académico mediante becas obtenidas en organismos internacionales ${ }^{4}$.

Todas estas acciones fueron confluyendo para el cuestionamiento del plan de estudios y la entrada en vigencia de un nuevo plan en 1963. Este sufrió un giro a favor de las ciencias sociales y de la "enseñanza de la metodología profesional" (ALVARIÑO et al., 1965, p. 11), considerada característica de la profesión del Servicio Social. Las materias que incluyó el nuevo plan de estudios fueron: "Antropología, Economía y Desarrollo, Teoría y Técnicas Administrativas, Cooperativismo, Política Social y Planificación de Grupo". La enseñanza práctica (Servicio Social Aplicado) concede especial importancia al conocimiento de la realidad social a través de unidades de investigación de áreas fundamentales como el campo de la Salud, del Trabajo y Salario, de la Vivienda, etc. (EDITORIAL, 1966).

Estos cambios se enfrentaron al proceso existente desde 1966, en la Universidad de Chile y conocido de "Reforma Universitaria" en 1968. Bajo una creciente movilización social y el gobierno democratacristiano de Eduardo Frei, cuyo programa gubernamental incentivó la participación social, la promoción popular y la Reforma Agraria, las Universidades chilenas comenzaron a problematizar la situación social, económica y política del país.

Los diagnósticos en la época, acerca de la situación de la Universidad, daban cuenta de una crítica situación social del país y de la universidad en particular. Problemas de modernización, de eficiencia, democratización y elitización eran algunos de los que se mencionaban y se exigía resolver (GARRETÓN; MARTINEZ, 1985, p. 11-31). La Reforma Universitaria adquirió mayor efervescencia y conflictividad debido al proceso político-social vivido paralelamente en el país, con la llegada, al gobierno, del socialista Salvador Allende como presidente de la república, en 1970, y de la Unidad Popular (cabe decir que la Unidad Popular - UP - fue una coalición electoral formada en 1969 y constituida por partidos chilenos de izquierda: Socialista, Comunista, Movimiento de Acción Popular Unitaria, Radical, de Izquierda Radical y Acción Popular Independiente).

Los profesionales del Servicio Social en América Latina, desde mediados de la década de 1960, comenzaron un proceso de problematización de la profesión y de la formación. En Chile, este movimiento cristalizó a partir de 1968. Cabe decir que, al interior de la Escuela Dr. Lucio

\footnotetext{
${ }^{4}$ En 1956 la Sra. Reyna Israel tuvo la oportunidad de estudiar en Puerto Rico y EEUU. Varios docentes pudieron participar en eventos como: Congresos Panamericanos en Cuba en 1959, en Costa Rica el 1960, la Conferencia y Congreso internacional de Escuelas en Italia el 1961, la Conferencia Internacional y el Congreso de Escuelas de Servicio Social, ambos en Brasil el año 1962 (EDITORIAL, 1966, p. 32).
} 


\section{ReVl|stg am Pallt}

\} EDUCACIÓN DEL TRABAJO SOCIAL EN CHILE - MOLINA, P. V. \}

DOI: $10.12957 /$ REP.2019.45217

Córdova, el movimiento reconceptualizador se expresó en 1968, a través de la apertura de una línea de investigación en la Escuela, encaminada a reflexiona acerca de los aspectos estudiados por el movimiento de reconceptualización ${ }^{5}$. Las críticas del movimiento mencionaban que los profesionales trabajaban con problemas a escala local, pero el nivel de análisis de las causas de estos no consideraba la dimensión macrosocial.

Asimismo, la intervención se reducía a la esfera asistencial. Dentro del quehacer profesional, los problemas se consideraban relacionados a las personas, los grupos o la comunidad, de manera aislada de la estructura social. Por lo tanto, en la medida que los sujetos, los grupos y comunidades los enfrentaban y superaban, sería posible la integración a la sociedad. Otras críticas realizadas por los estudiantes de Servicio Social fueron dirigidas a los métodos que usaba, la profesión, para la intervención. Señalaban que estos métodos (caso, grupo y desarrollo de la comunidad), habían sido importados desde los países desarrollados, sin realizar las adecuaciones y reflexiones pertinentes para el contexto latinoamericano (especialmente acerca de las necesidades de la sociedad chilena):

[en] los objetivos de la técnica de Caso se encuentran conceptos contrarios al cambio como: 'reajustar al individuo al medio y que éste sea el propio agente directo de su 'adaptación'. Junto a esto se observa que fundamentalmente el caso social está determinado por conflictos o problemas de personalidad que se traducen en un determinado comportamiento o por factores culturales o sociales de la realidad social. En general en esta técnica se ha enfatizado la importancia del primer elemento, excluyendo el análisis de las causas que generan una situación problema y que derivan especialmente de las deficiencias en la estructura social' (Allende, Mario, et. al.; 1969: 291). Asimismo, la crítica al método de grupo (concebido como un proceso socio-educativo cuyo objetivo es el desarrollo de la personalidad y la adaptación social de los individuos a través de asociaciones voluntarias que se constituyen en medios para alcanzar fines socialmente deseables (Newsteter en Allende, Mario, et. al.; 1969: 292) se traza en relación a su insuficiencia para lograr efectiva participación popular, centrándose en un conjunto de individuos en sí mismos, a su personalidad y grupo, desarticulada de la política nacional. (ALLENDE, 1969, p. 292).

Por otro lado, la crítica se extendió a las instituciones de práctica existentes para la formación de los estudiantes. Se afirmaba que estos organismos, principalmente gubernamentales, planteaban límites claros al quehacer transformador del profesional, porque respondían a las orientaciones de las políticas sociales de los gobiernos de turno. Es decir, las

${ }^{5}$ Véase más en: "Un análisis crítico de la teoría del Servicio Social" (RUZ, 1968), "Servicio Social, un instrumento de Desarrollo" (ALLENDE et al., 1969), "Un análisis crítico de la metodología del Servicio Social” (HERNANDEZ, 1970), "Un análisis crítico de la práctica del Servicio Social" (MENDEZ et al., 1970) y "Los determinantes 


\section{ReVIStG PMI PDUtD}

\} EDUCACIÓN DEL TRABAJO SOCIAL EN CHILE - MOLINA, P. V. \}

DOI: $10.12957 /$ REP.2019.45217

prácticas institucionales jugaban en contra de los nuevos objetivos que la profesión fue asumiendo desde la segunda mitad de 1960: constituirse en agente de cambio de las estructuras de la sociedad chilena.

Era una crítica que afectaba a la ideología, la teoría y metodología de la profesión. La noción de práctica social o praxis social, sustentada desde fundamentos marxistas, fue la que adquirió relevancia para pensar un nuevo tipo de Servicio Social, comprometido con la transformación social.

En 1970, con la llegada de la UP, la crítica institucional levantada desde el Servicio Social fue aminorada, porque, para este nuevo gobierno, las instituciones y organismos del Estado eran un pilar central para avanzar en la transformación social que Chile requería.

Situados en el marco de referencia que da la ascensión al poder de un gobierno popular, se estima que la Escuela no puede permanecer al margen de esta perspectiva de cambio básico de estructuras que se abre al país. El compromiso de la disciplina con los cambios será real, en la medida en que ella participe activamente -desde el punto de vista de su quehacer- en todas aquellas medidas y acciones que el gobierno popular impulse para llevar a cabo sus tareas, aportando todos aquellos elementos necesarios que contribuyan a la realización y el éxito de ellas. (MENDEZ et al., 1970, p. 84).

El gobierno de la UP definía las características estructurales de la economía chilena de carácter dependiente y monopólico. Así, a partir de la llegada al gobierno de Salvador Allende, se comenzó un proceso de transformación económica de Chile. Para ello, la creación de un Área Social dominante, dentro de la economía (o lo que normalmente era definido como mundo empresarial e industrial) fue prioridad.

La lucha por desplazar al imperialismo, los monopolios y el latifundio de los centros de la decisión económica, tuvo importancia decisiva en la batalla general por el poder en Chile. Para el gobierno de Salvador Allende, el Área Social dominante era capaz de dirigir la economía en su conjunto, aumentando la producción de bienes y el control de aparato productivo.

La creación del Área Social de las empresas, es decir, empresas dirigidas por el Estado y los trabajadores era considerado un instrumento decisivo en la transición hacia la construcción socialista. Entonces, la tarea imprescindible era generar una amplia participación de los trabajadores en este proceso, porque permitía - como se señalaba en la época - experimentar formas de democracia proletaria.

En este contexto, el Asistente Social podía ayudar al proceso de participación de los trabajadores en las Empresas del Área Social. Así, algunas de las tesis y memorias realizadas por Asistentes Sociales se identificaron críticamente con los desafíos políticos y económicos que el gobierno de la UP definía. A modo de ejemplo, los estudios alertaban acerca de los procesos 


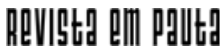

\} EDUCACIÓN DEL TRABAJO SOCIAL EN CHILE - MOLINA, P. V. \}

DOI: $10.12957 /$ REP.2019.45217

de burocratización de los organismos de participación de los trabajadores, al interior de estas empresas. Problematizaban las normas básicas de participación impulsadas por el gobierno. En ese sentido, las investigaciones de los estudiantes de Servicio Social también tensionaron las políticas y acciones del gobierno, para favorecer la participación de los trabajadores en este tipo de empresas.

Entre 1970 y 1973, los estudiantes de Servicio Social (especialmente de la Universidad de Chile) asumieron que la profesión aportaba en la construcción del proyecto histórico de la UP, porque sus funciones estaban dirigidas a los sectores populares para que estos pudieran participar en el poder político. Identificaban, claramente, que la función fundamental del profesional era la educativa. Con ella se podía crear conciencia crítica en cada persona, para enfrentar sus problemas. Es decir, crear, en cada persona, la necesidad de cambiar la situación social problemática y prepararlas para enfrentar el proceso de participación en su comunidad para, así, avanzar en construir una nueva sociedad.

\section{Neoliberalismo y contradicciones del Trabajo Social chileno: 1973-2013}

El golpe de Estado, el 11 de Septiembre de 1973, cambió radicalmente la historia de Chile. Los muertos, exiliados y desaparecidos dan cuenta de la dictadura más sangrienta del cono sur en América Latina. La Dictadura permitió que Chile fuera el laboratorio del Neoliberalismo en el mundo. A las políticas represivas, de exterminio de la izquierda chilena y de la organización colectiva, se sumaron políticas económicas y sociales que devastaron Derechos Sociales ganados por las masas populares tras años de luchas. Los cambios fueron estructurales: la privatización de los servicios de salud, educación, vivienda y de los recursos naturales, la anulación de políticas sociales universales y la focalización en sectores de extrema pobreza, entre otros cambios, fueron amparados por una nueva Constitución - la de 1980.

El Servicio Social sufrió los golpes de la Dictadura, tanto por, la muerte de varios estudiantes, el cierre de la Escuela de Servicio Social Dr. Lucio Córdova (comenzado en 1973 y realizado efectivamente en 1980), la eliminación de las sedes regionales de la universidad, la transformación de la malla curricular, como por la exoneración de profesores y expulsión de estudiantes.

Todo ello impactó en las escuelas de Servicio Social del país. En la orientación de las mallas curriculares de las escuelas de Trabajo Social (como pasaron a llamarse), pasa a tener prioridad la formación tecnológica, desideologizada, asistencialista y paternalista. Desde la dimensión de la intervención, ligado al quehacer profesional al interior de las instituciones 


\section{ReVIStG PMI PDUtD}

\} EDUCACIÓN DEL TRABAJO SOCIAL EN CHILE - MOLINA, P. V. \}

DOI: $10.12957 /$ REP.2019.45217

del Estado, la atención individual se transformó en prioritaria, no así la de los grupos y comunidades. En paralelo, se gestó fuera de las instancias del Estado, un colectivo de Asistentes Sociales ligado a la defensa de los Derechos Humanos, la promoción de la participación social y la democratización de la sociedad.

La lucha y movilización social contra la dictadura militar de Pinochet, durante la década de 1980, permitió que se pactara la realización de un plebiscito para decidir la continuidad o no de Pinochet en el poder. Este plebiscito fue ganado por la oposición a la dictadura, en 1988. Desde 1990 comenzó el proceso de reconstrucción de la democracia en el país, que mantuvo en el gobierno a la Concertación de Partidos por la Democracia $^{6}$, hasta el 2009. Dichos gobiernos mantuvieron el modelo económico Neoliberal implantado por la Dictadura, pero sumaron - entre otras cosas un carácter social a su gestión: la preocupación por la superación de la pobreza y la equidad social, para disminuir las desigualdades profundas dejadas por el régimen militar.

En este contexto, la formación universitaria de Trabajo Social asumió estas preocupaciones, es decir, apoyar fuertemente las políticas sociales elaboradas por estos gobiernos. La formación en gestión, elaboración de proyectos, medición de la pobreza, además de una fuerte formación en Ciencias Sociales (Sociología, Antropología, Psicología, Filosofía, Estadística, Investigación Social, Derecho) fue prioridad para impulsar y consolidar la superación de la pobreza, la ampliación de la focalización hacia otros sujetos sociales, la participación social de la comunidad en la definición de sus problemáticas, entre otros.

A comienzos de la década de 1990, existía en Chile solo una universidad privada que impartía la carrera de Trabajo Social. Después de 25 años, la proliferación de la educación privada avanzó a un nivel insospechado, superando la oferta - en Trabajo Social - entregada por las universidades públicas chilenas. Actualmente en Chile, la formación de Trabajo Social la imparten 16 universidades públicas y 24 universidades privadas, con sedes en regiones a lo largo del país, las que en su totalidad ofrecen más de 200 programas de formación. Las mallas curriculares poseen diversos enfoques y énfasis. A pesar de este escenario, aún no se logra levantar, a nivel nacional de las escuelas, un cuestionamiento a la excesiva proliferación de programas de formación universitaria con consecuencias en el quehacer profesional.

Para el año 2013, las vacantes correspondientes a la oferta formativa de nivel superior en Trabajo Social es de aproximadamente 21.000 cupos. La calidad de estos programas de formación son evaluados por

\footnotetext{
${ }^{6}$ Esta coalición estaba conformada principalmente por la alianza demócrata cristiana y socialista, después del abandono de parte de los socialistas, del marxismo y su orientación de los años 70. Los Presidentes fueron: Andrés Aylwin (1990-1994), Eduardo Frei Ruiz Tagle (1994-2000), Ricardo Lagos (2000-2006), Michelle Bachelet (2006-2010)
} 


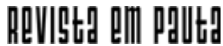

\} EDUCACIÓN DEL TRABAJO SOCIAL EN CHILE - MOLINA, P. V. \}

DOI: $10.12957 /$ REP.2019.45217

agencias de acreditación, externas a las propias universidades. A partir del proceso de acreditación al que deben someterse las escuelas de Trabajo Social en Chile, los datos muestran que las universidades públicas se encuentran acreditadas en mayor cantidad que las escuelas impartidas por universidades privadas, como se observa en el Gráfico 2.

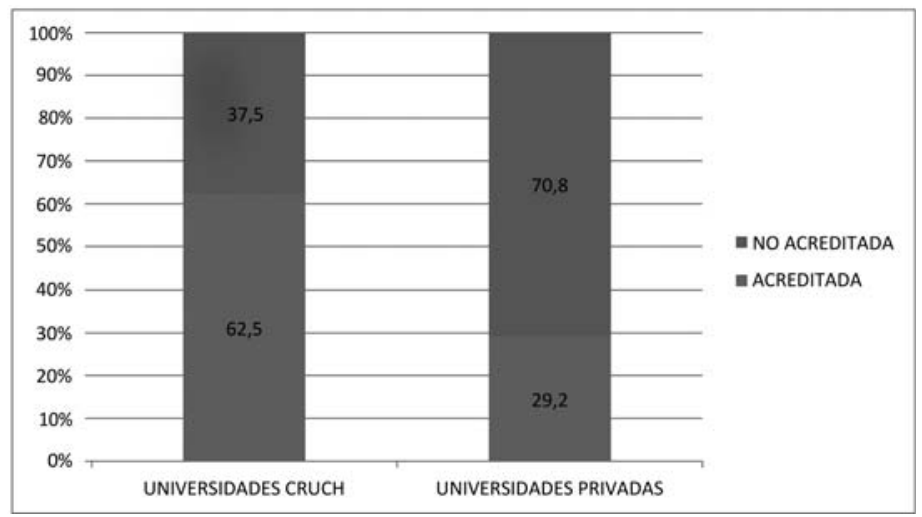

Elaboración basada en fuentes de la Comisión Nacional de Acreditación Chile (2013).

Desde la década de 2000 se inicia en Chile la formación de postgrado, especialmente a nivel de Magíster en Trabajo Social, asociado al proceso de expansión de la oferta educacional anteriormente mencionado. Lo anterior conlleva la expansión de espacios de desempeño profesional en instituciones del Estado, Tercer Sector (ONGs, Fundaciones sin fines de lucro, etc.) y Empresas.

Actualmente existen 10 instituciones de formación académica de postgrado en el país que apuntan a reflexionar acerca de temáticas o áreas vinculadas a la profesión, es decir, desde un énfasis profesionalizante. Esto tiene mucha relación con la preponderancia que dicho énfasis ha tenido para articular formación y empleabilidad.

La oferta de postgrado (magíster) se orienta a mejorar competencias en temáticas específicas, como en superación de la pobreza, intervención social en drogas, familia, jóvenes, adultos mayores, mujeres, en comunidades, con énfasis en interculturalidad, gestión, mediación jurídica, etc.. Ninguno de estos programas establece puentes con un desarrollo reflexivo y riguroso desde un debate disciplinario del Trabajo Social, para con esas temáticas.

El desafío, en este escenario, es lograr construir un campo de reflexión de postgrado en donde se observe la capacidad de articular conocimiento disciplinario con líneas de acción, en la perspectiva de que Trabajo Social deje de concebirse como un mero ejecutor o "técnico" de políticas sociales. 


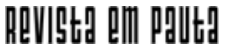

\} EDUCACIÓN DEL TRABAJO SOCIAL EN CHILE - MOLINA, P. V. \}

DOI: $10.12957 /$ REP.2019.45217

\section{Palabras finales}

Hasta aquí, el lector habrá seguido el devenir de la formación del Servicio Social en Chile, primer país en América Latina en aceptar y definir un área profesional para la educación especializada. Dicho devenir no estuvo ajeno a las influencias políticas y sociales desarrolladas a nivel nacional del siglo XX al XXI. En este sentido, la profesión siguió los vaivenes y desafíos puestos por los gobiernos y por el movimiento social en cada época.

Hoy los desafíos sociales, económicos, culturales y políticos en Chile ponen, a la formación universitaria y al ejercicio profesional del Trabajo Social, en una disyuntiva. Se mantiene en el horizonte heredado de la dictadura, o aporta en profundizar las demandas del movimiento social (levantado a partir del 2011), que exige una educación y salud como un derecho social.

En definitiva, hoy la urgencia del debate al interior de la formación profesional en Chile se impone también por la urgencia de trasformar y avanzar hacia una estructura social más justa. Diversos sectores sociales (estudiantes, mapuches, trabajadores, pescadores, mujeres, etc), en los últimos tres años han tenido la claridad y fuerza para decir que el experimento neoliberal en Chile, ha sido un fracaso.

Las Escuelas de Trabajo Social en Chile, ipueden ponerse a tono con los nuevos tiempos? ¿Pueden generar una formación que permita a sus estudiantes y futuros profesionales posicionarse reflexivamente y a favor de cambios que clama la sociedad chilena? Este escenario, desafía los compromisos, los valores, sentidos, certezas, teorías y prácticas del Trabajo Social en Chile. Por esto, revisar y repensar 90 años de historia profesional, se hace más necesario que nunca. 


\section{ReVIStg a d P p putt}

\} EDUCACIÓN DEL TRABAJO SOCIAL EN CHILE - MOLINA, P. V. \}

DOI: $10.12957 /$ REP.2019.45217

\section{Referencias}

ALVARIÑO, P.; ISRAEL, R.; MORENO, C. et al. Las escuelas de Servicio Social y la política social. Revista Servicio Social, Santiago, v. 3, 1965.

CARDENAS, L. Algunas características del Servicio Social. In: MATUS, T.; FORTTES, A.; AYLWIN, N. La reinvención de la memoria. Revista Servicio Social, Santiago, año 2, v. 1, 2004.

CERECEDA, X. Estudio de la situación ocupacional de los asistentes sociales de las últimas seis promociones de las escuelas de Servicio Social de la Universidad de Chile, Santiago-Valparaiso-Antofagasta. Memoria de prueba para optar al título de Asistente Social. Escuela de Servicio Social. Santiago. Universidad de Chile. Santiago. Chile. 1968.

COMISIÓN NACIONAL DE ACREDITACIÓN EN CHILE. Disponible en: http:/ /www.cnachile.cl/. Accedido en: 7 ago.

EDITORIAL. Historia de la Escuela de Servicio Social "Dr. Lucio Córdova" de la Universidad de Chile". Revista Servicio Social, Santiago, v. 4, 1966.

GARRETÓN, M.; MARTÍNEZ, J. La reforma en la Universidad de Chile. Tomo III. Santiago: Sur Ediciones, 1985.

GARIB, P. Evolución y perspectivas del Desarrollo de la Comunidad en Chile. Tesis de grado para optar al título de Servicio Social. Escuela de Servicio Social. Universidad de Chile. Santiago. Chile. 1969.

ILLANES, M. El cuerpo y la sangre de la política. La construcción histórica de las visitadoras sociales (1887-1940). Santiago: Lom Ediciones, 2007.

MENDEZ, J.; PIZARRO, M. et al. Análisis crítico y bases para la reformulación de la práctica del servicio social. Memoria para optar al título de Asistente Social. Santiago: Escuela de Servicio Social, 1970.

MATUS, T.; AYLWIN, N.; FORTTES, A. La de la Memoria. Santiago: Publicaciones UC, 2004.

MILOS, P. El Frente Popular. Santiago: Lom Ediciones, 2008.

ROMÁN, C.; ZULOAGA, M. Metodología básica de Servicio Social. Memoria para optar al título de Servicio Social. Santiago: Escuela de Servicio Social Dr. Lucio Córdova, 1970.

SAND, R. Las escuelas de Servicio Social. In: MATUS, T.; FORTTES, A.; AYLWIN, N. La reinvención de la memoria. Santiago: Pontificia Universidad Católica de Chile, 2004. UNIVERSIDAD DE CHILE. Anales de la Facultad de Ciencias Jurídicas y Sociales, v. 3, n. 3, 1962. Disponible en: http:// www.analesderecho.uchile.cl/index.php/ACJYS/article/viewArticle/10274/ 10330. Accedido en: 06 agosto 2013. 


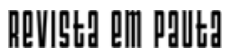

\} EDUCACIÓN DEL TRABAJO SOCIAL EN CHILE - MOLINA, P. V. \}

DOI: 10.12957/REP.2019.45217

DOI: $10.12957 /$ rep.2019.45217

Recebido em 26 de abril de 2019.

Aprovado para publicação em 02 de maio de 2019.

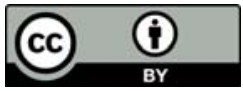

A Revista Em Pauta: Teoria Social e Realidade Contemporânea está licenciada com uma Licença Creative Commons Atribuição 4.0 Internacional. 